\title{
DEFORMATIONS OF VECTOR FIELDS AND HAMILTONIAN VECTOR FIELDS ON THE PLANE
}

\author{
NICO VAN DEN HIJLIGENBERG, YOURI KOTCHETKOV, AND GERHARD POST
}

\begin{abstract}
For the Lie algebras $L_{1}(H(2))$ and $L_{1}(W(2))$, we study their infinitesimal deformations and the corresponding global ones. We show that, as in the case of $L_{1}(W(1))$, each integrable infinitesimal deformation of $L_{1}(H(2))$ and $L_{1}(W(2))$ can be represented by a 2 -cocycle that defines a global deformation by means of a trivial extension. We also illustrate that all deformations of $L_{1}(H(2))$ arise as restrictions of deformations of $L_{1}(W(2))$.
\end{abstract}

\section{INTRODUCTION}

The investigation of deformations of nilpotent subalgebras $L_{1}(A)$ of graded infinite-dimensional Lie algebras $A$ is a new area of research. In the well-known work [1], A. Fialowski presents a complete description of the deformations of $L_{1}(W(1))$, the nilpotent part of the Witt algebra. This and similar works like [2] and [3] show that the properties of deformations of nilpotent parts are completely different from those of the corresponding graded Lie algebra. Usually $A$ is rigid while $L_{1}(A)$ has several nontrivial deformations which have a fairly simple algebraic description. Often the integrable infinitesimal deformations, these are the ones that can be extended to a global deformation, can be represented by a cocycle for which the Lie square equals zero. So, this cocycle defines a global deformation by simply putting the coefficient of $t^{p}$ in the deformed commutator equal to zero for all $p \geq 2$. We call this a trivial extension, as it means that the infinitesimal deformation is already a global deformation.

Our object is to investigate the deformations of $L_{1}(H(2 n))$, the nilpotent subalgebra of the Lie algebra of polynomial Hamiltonian vector fields in $(2 n)$ dimensional space, and $L_{1}(W(m))$, the nilpotent subalgebra of the Lie algebra of polynomial vector fields in $m$-dimensional space. In this paper, the cases $n=1$ and $m=2$ are studied. With the help of the Feigin-Fuks spectral sequence we compute infinitesimal deformations of these two Lie algebras. Necessary conditions for their integrability will be derived, and we shall prove that these conditions are also sufficient by constructing global deformations with the prescribed infinitesimal parts. We show that in all cases this can be done by means of a trivial extension, and that these global deformations are unique up

Received by the editor March 19, 1993 and, in revised form, December 7, 1993.

1991 Mathematics Subject Classification. Primary 17B56, 17B65, 17B66, 17B68, $17 \mathrm{~B} 70$.

Key words and phrases. Lie algebras, deformations.

Research of the second author was supported in part by NWO grant 07-30-001. 
to a transformation. Most interesting in the results concerning $L_{1}(H(2))$ is that all its deformations as well as their realizations can be regarded as restrictions of deformations and realizations of $L_{1}(W(2))$.

\section{DEFINITIONS AND PRELIMINARY REMARKS}

For the reader's convenience, we include here some definitions and remarks concerning deformations of Lie algebras. We assume that the reader is familiar with cohomology of Lie algebras (see, e.g., [6]). For deformations of a Lie algebra $L$, the cohomology of $L$ with coefficients in the adjoint representation is important. These cohomology groups are denoted by $H^{q}(L ; L)$ $(q=0,1,2, \ldots)$. A deformation (also called global deformation) of $L$ is usually written as

$$
[x, y]_{t}=[x, y]+\sum_{n=1}^{\infty} t^{n} \varphi_{n}(x \wedge y), \quad x, y \in L, \varphi_{n} \in C^{2}(L ; L) .
$$

Here, $t$ is a formal parameter (so that strictly speaking $[x, y]_{t} \in L[[t]]$ ). A deformation is called trivial if $[\cdot, \cdot]_{t}$ is the result of a change of basis $\beta: x \mapsto$ $x+\sum_{n=1}^{\infty} t^{n} \tau(x), \tau \in C^{1}(L ; L)$, see [6, page 35] for more details. Now $[\cdot, \cdot]_{t}$ should be a Lie algebra. The requirements that the deformed Lie product should be bilinear and anti-symmetric is reflected in $\varphi_{n} \in C^{2}(L ; L)$. The requirement that Jacobi's identity should be satisfied for the coefficient of $t^{n}$ leads to

$$
\mathrm{d}\left(\varphi_{n}\right)+\frac{1}{2} \sum_{i=1}^{n-1} M\left(\varphi_{i}, \varphi_{n-i}\right)=0
$$

where

$$
\begin{aligned}
M\left(\varphi_{i}, \varphi_{j}\right)(x \wedge y \wedge z)= & \varphi_{i}\left(\varphi_{j}(x \wedge y) \wedge z\right)+\varphi_{i}\left(\varphi_{j}(y \wedge z) \wedge x\right) \\
& +\varphi_{i}\left(\varphi_{j}(z \wedge x) \wedge y\right)+\varphi_{j}\left(\varphi_{i}(x \wedge y) \wedge z\right) \\
& +\varphi_{j}\left(\varphi_{i}(y \wedge z) \wedge x\right)+\varphi_{j}\left(\varphi_{i}(z \wedge x) \wedge y\right) .
\end{aligned}
$$

We will call $M\left(\varphi_{i}, \varphi_{j}\right)$ the product of $\varphi_{i}, \varphi_{j}$, and $M\left(\varphi_{1}, \varphi_{1}\right)$ the Lie square of $\varphi_{1}$, and $M\left(\varphi_{1}, \varphi_{2}\right)$ the Massey cube of $\varphi_{1}$.

From equation (1), one can see several things. The first is that $d\left(\varphi_{1}\right)=0$, so that $\varphi_{1} \in Z^{2}(L ; L)$. This $\varphi_{1}$ is called the infinitesimal part of the deformation. Now for trivial deformations one can prove that $\varphi_{1} \in B^{2}(L ; L)$. Hence, to study the possible interesting infinitesimal deformations, one needs to calculate $H^{2}(L ; L)$.

This is always the basis for constructing (nontrivial) deformations. However, not every infinitesimal deformation $\varphi_{1}$ can be extended to a deformation (said differently: can be integrated). For this, one needs to find a sequence $\left(\varphi_{n}\right)$ $(n=2,3, \ldots)$ satisfying equation (1). This is a difficult problem. For example, suppose $\varphi_{1}$ is given. Then one tries to construct $\varphi_{2}$. For this, one needs $M\left(\varphi_{1}, \varphi_{1}\right)=0$ as an element of $H^{3}(L ; L)$. In this case we say that the product is trivial, and the obstruction is solvable. If so, $\mathrm{d}\left(\varphi_{2}\right)$ is fixed, but there is still freedom in $\varphi_{2}$ itself. This freedom cannot be fixed: $\varphi_{2}$ appears in all next equations of (1), and depending on the choice of $\varphi_{2}$, these equations may or may not be solvable. Moreover, different choices of $\varphi_{2}$ may lead to different 
deformations which cannot be transformed into one another. This happens for sure when for two extensions $\left(\varphi_{n}\right)$ and $\left(\varphi_{n}^{\prime}\right)$ with $\varphi_{m}=\varphi_{m}^{\prime}$ for $m=$ $1,2, \ldots, i-1$, we have that $\varphi_{i}-\varphi_{i}^{\prime}$ is nonzero as an element of $H^{2}(L ; L)$. Conversely, if $\varphi_{m}=\varphi_{m}^{\prime}$ for $m=1,2, \ldots, i-1$ and $\varphi_{i}-\varphi_{i}^{\prime}$ is zero as an element of $H^{2}(L ; L)$, then one can find a transformation such that $\varphi_{i}=\varphi_{i}^{\prime}$. Hence one can try to match $\left(\varphi_{n}\right)$ and $\left(\varphi_{n}^{\prime}\right)$ recursively.

In the sequel we will do the following. We will find infinitesimal deformations for $H(2)$ and $W(2)$ and check in what cases these infinitesimal deformations can be extended to a deformation. At first we will satisfy ourselves with the construction of one such an extension; at the end of the paper we will show that this leads to all homogeneous deformations.

\section{Deformations of $L_{1}(H(2))$}

2.1. The cohomology space $H^{2}\left(L_{1} ; L_{1}\right)$. We consider $H(2)$, i.e., the Lie algebra of polynomial Hamiltonian vector fields on the plane. This Lie algebra is isomorphic to the Poisson algebra $\mathbb{C}[p, q]$ modulo its center. An isomorphism can be given by mapping $p^{k} q^{\ell} \in \mathbb{C}[p, q]$ to $k p^{k-1} q^{\ell} \partial_{q}-\ell p^{k} q^{\ell-1} \partial_{p} \in W(2)$. For the sake of convenience we shall make no distinction between these elements. So we view $H(2)$ both as a Lie subalgebra of $W(2)$ as well as a quotient algebra of the Poisson algebra. A basis of this quotient algebra is given by $\left\{p^{k} q^{\ell} \mid k+\ell>0 ; k, \ell \geq 0\right\}$, and the commutator satisfies

$$
\left[p^{k} q^{\ell}, p^{m} q^{n}\right]=(k n-\ell m) p^{k+m-1} q^{\ell+n-1} \quad(\bmod 1) .
$$

On $H(2)$ there exist two gradings, which are denoted by deg (degree) and wg (weight). With respect to these gradings the monomials are homogeneous: $\operatorname{deg}\left(p^{k} q^{\ell}\right)=k+\ell-2$ and $\operatorname{wg}\left(p^{k} q^{\ell}\right)=k-\ell$. For all $k$ in $\mathbb{Z}$ we define $L_{k}=L_{k}(H(2))$ to be the Lie subalgebra of $H(2)$ generated by the elements of degrec greater than or equal to $k$. The two gradings on $H(2)$ induce a bigraded structure on the cohomology groups. Let $H_{(d, w)}^{2}\left(L_{1} ; L_{1}\right)$ be the cohomology group of cocycles $c$ such that

$$
\begin{gathered}
\operatorname{deg}\left(c\left(x_{1} \wedge x_{2}\right)\right)=d+d_{1}+d_{2} \quad \text { if } \operatorname{deg}\left(x_{i}\right)=d_{i} \text { and } \\
\operatorname{wg}\left(c\left(x_{1} \wedge x_{2}\right)\right)=w+w_{1}+w_{2} \quad \text { if } \operatorname{wg}\left(x_{i}\right)=w_{i} .
\end{gathered}
$$

We shall discuss deformations of the nilpotent Lie subalgebra $L_{1}$. For computational reasons we need to restrict ourselves to the investigation of deformations of $L_{1}$ that are homogeneous with respect to the degree and weight. The computation of $H_{(d, w)}^{2}\left(L_{1} ; L_{1}\right)$ can be performed by using the Feigin-Fuks spectral sequence. The initial term is (see also [4])

$$
E_{1}^{p, q}=\bigoplus_{w^{\prime}} H_{\left(-p, w^{\prime}\right)}^{p+q}\left(L_{1}\right) \otimes\left(L_{1}\right)_{\left(d+p, w-w^{\prime}\right)}
$$

From [5] we know that $H_{(d)}^{2}\left(L_{1}\right)=0$ for all $d \notin\{-2,-3,-4\}$. Thus, from the spectral sequence we can conclude that nonzero cohomology groups can only occur for $d \geq-3$. By means of computer calculations we obtained the following result. 
Theorem 1. There holds

$$
\operatorname{dim} H_{(d, w)}^{2}\left(L_{1} ; L_{1}\right)= \begin{cases}1, & d=-2, \quad w \in\{-4,-2,0,2,4\}, \\ 3, & d=-1, \quad w \in\{-3,-1,1,3\}, \\ 0 \quad \text { in all other cases } \text { with } d \leq 0 .\end{cases}
$$

We did not investigate the case $d>0$. The motivation for this is that, since $H(2)$ is rigid, we do not expect nontrivial deformations of positive degree to be present.

2.2. Global deformations. For the case $d=-2$, it turned out that the Lie square of the cocycles is nontrivial. Hence there is no global deformation corresponding to these cocycles. Let us consider the case $d=-1$. Since $w=3$ and $w=-3$, respectively $w=1$ and $w=-1$, convert to each other after interchanging $p$ and $q$, we consider only $w=1$ and $w=3$.

Case 1: $\mathbf{w}=1$. The cohomology space $H_{(-1,1)}^{2}\left(L_{1} ; L_{1}\right)$ is generated by $c_{1}, c_{2}$ and $c_{3}$ with

$$
\left\{\begin{array}{l}
c_{1}\left(p q^{2} \wedge p^{k} q^{\ell}\right)=(k-\ell) p^{k} q^{\ell},(k, \ell) \neq(1,2), \\
c_{2}\left(p^{2} q \wedge p^{k} q^{\ell}\right)=-2 \ell p^{k+1} q^{\ell-1},(k, \ell) \neq(2,1), \\
c_{3}\left(p q^{2} \wedge p^{k} q^{\ell}\right)=-(k+\ell-2) p^{k} q^{\ell},(k, \ell) \neq(1,2) .
\end{array}\right.
$$

These elements $c_{i}$ are induced by elements $b_{i} \in C^{1}\left(L_{1} ; \overline{L_{0}}\right)$, where $\overline{L_{0}}$ is the Lie subalgebra of $W(2)$ given by $L_{0} \oplus\left\langle p \partial_{p}+q \partial_{q}\right\rangle$. Let us use the notation $x \mapsto y$ for the element $b$ in $C^{1}\left(L_{1} ; \bar{L}_{0}\right)$, with $b(x)=y$ and $b(z)=0$ for all other monomials $z$. One easily checks that $c_{i}=\mathrm{d}\left(b_{i}\right)$, where

$$
b_{1}=p q^{2} \mapsto p q, \quad b_{2}=p^{2} q \mapsto p^{2} \quad \text { and } \quad b_{3}=p q^{2} \mapsto\left(p \partial_{p}+q \partial_{q}\right) .
$$

The fourth candidate, $\mathrm{d}\left(q^{3} \mapsto q^{2}\right)$, is linearly dependent in $H_{(-1,1)}^{2}\left(L_{1} ; L_{1}\right)$. This follows from the following observation. We take $\alpha \in C_{(-1,1)}^{0}\left(L_{1} ; H(2)\right)$ given by $\alpha=1 \mapsto p$ and compute

$$
\mathrm{d}(\alpha)=3\left(q^{3} \mapsto q^{2}\right)+2 b_{1}+b_{2}+\sum_{k+\ell \geq 4} \ell\left(p^{k} q^{\ell} \mapsto p^{k} q^{\ell-1}\right) .
$$

By applying the coboundary operator $\mathrm{d}$ and using $\mathrm{d}^{2}=0$, we obtain

$$
3 \mathrm{~d}\left(q^{3} \mapsto q^{2}\right)+2 c_{1}+c_{2} \in B_{(-1,1)}^{2}\left(L_{1} ; L_{1}\right) .
$$

This expresses the linear dependency in $H_{(-1,1)}^{2}\left(L_{1} ; L_{1}\right)$.

For the linear combination $c=\alpha_{1} c_{1}+\alpha_{2} c_{2}+\alpha_{3} c_{3}$ we calculated the obstructions. The Lie square is trivial for all values of the parameters, but the Massey cube is trivial only if $\alpha_{2}=0$ or if $\alpha_{1}=-\alpha_{3}$. The first case, $\alpha_{2}=0$, describes a plane of cocycles that obviously have a trivial extension. It has the following geometrical interpretation. Define $\varphi: L_{1} \longrightarrow \overline{L_{0}}$ by $\varphi=$ id $-t b$ with $b=\alpha_{1}\left(p q^{2} \mapsto p q\right)+\alpha_{3}\left(p q^{2} \mapsto\left(p \partial_{p}+q \partial_{q}\right)\right)$. For all values of $\alpha_{1}$ and $\alpha_{3}$, $\varphi\left(L_{1}\right)$ is a subalgebra in $\overline{L_{0}}$, and one easily verifies that the deformation given by $c$ for $\alpha_{2}=0$ can be written as $[x, y]_{t}=\varphi^{-1}([\varphi(x), \varphi(y)])$. So this plane describes what can be called embedded deformations. 
The second plane is generated by the cocycles $c_{1}-c_{3}$ and $c_{2}$. Instead of $c_{2}$ we choose a different representative, namely $\tilde{c_{2}}=\mathrm{d}\left(\tilde{b}_{2}\right)$, with

$$
\begin{aligned}
\tilde{b_{2}}= & \sum_{\ell \geq 3} \frac{\ell(\ell-3)}{\ell-1}\left(q^{\ell} \mapsto q^{\ell-1}\right)+\sum_{\ell \geq 2}(\ell-2)\left(p q^{\ell} \mapsto p q^{\ell-1}\right) \\
& +\sum_{k \geq 2, \ell \geq 1} \ell\left(p^{k} q^{\ell} \mapsto p^{k} q^{\ell-1}\right) .
\end{aligned}
$$

Note that $\left(b_{2}-\widetilde{b_{2}}\right) \in C^{1}\left(L_{1} ; L_{1}\right)$, so that $c_{2}$ and $\widetilde{c_{2}}$ are indeed cohomologous. We prove that the Lie square of $\tilde{c_{2}}$ is equal to zero, and that the product of $\tilde{c_{2}}$ and $c_{1}-c_{3}$ equals zero. From this, the cocycles in the second plane also define a global deformation by means of a trivial extension. In order to prove that $M\left(\tilde{c_{2}}, \tilde{c_{2}}\right)=0$, one just has to check several cases. Here we only write out two of them; all other cases can be handled similarly. First we write down the nonzero actions of $\tilde{c_{2}}$ :

$$
\begin{array}{ll}
\tilde{c_{2}}\left(q^{\ell} \wedge p q^{m}\right) & =\frac{-2 \ell(\ell+m-3)}{(\ell+m-2)} q^{\ell+m-2}, \\
\tilde{c_{2}}\left(q^{\ell} \wedge p^{m} q^{n}\right) & =-2 \ell m p^{m-1} q^{\ell+n-2} \quad(m>2), \\
\tilde{c_{2}}\left(p q^{\ell} \wedge p q^{m}\right) & =2(m-\ell) p q^{\ell+m-2}, \\
\tilde{c_{2}}\left(p q^{\ell} \wedge p^{m} q^{n}\right) & =2(m+n-\ell m) p^{m} q^{\ell+n-2} \quad(m \geq 2) .
\end{array}
$$

We consider the cases

(i) $\frac{1}{2} M\left(\tilde{c_{2}}, \tilde{c_{2}}\right)\left(q^{\ell} \wedge p q^{m} \wedge p q^{n}\right)$

$$
\begin{aligned}
= & \frac{-2 \ell(\ell+m-3)}{(\ell+m-2)} \tilde{c_{2}}\left(q^{\ell+m-2} \wedge p q^{n}\right) \\
& \quad-2(m-n) \tilde{c_{2}}\left(p q^{m+n-2} \wedge q^{\ell}\right)+\frac{2 \ell(\ell+n-3)}{(\ell+n-2)} \tilde{c_{2}}\left(q^{\ell+n-2} \wedge p q^{m}\right) \\
= & \frac{(m+\ell+n-5)}{(m+\ell+n-4)}(4 \ell(\ell+m-3)-4 \ell(m-n)-4 \ell(\ell+n-3)) q^{m+n+\ell-4}=0 .
\end{aligned}
$$

(ii) $\frac{1}{2} M\left(\tilde{c_{2}}, \tilde{c_{2}}\right)\left(p q^{\ell} \wedge p q^{m} \wedge p q^{n}\right)$

$$
\begin{aligned}
& =2(m-\ell) \tilde{c_{2}}\left(p q^{m+\ell-2} \wedge p q^{n}\right) \\
& \quad+2(n-m) \tilde{c_{2}}\left(p q^{m+n-2} \wedge p q^{\ell}\right)-2(n-\ell) \tilde{c_{2}}\left(p q^{n+\ell-2} \wedge p q^{m}\right) \\
& =2\{(m-\ell)(n-m-\ell+2)+2(n-m)(\ell-m-n+2) \\
& \quad-2(n-\ell)(m-n-\ell+2)\} p q^{n+\ell+m-4}=0 .
\end{aligned}
$$

The proof of $M\left(\tilde{c_{2}}, c_{1}-c_{3}\right)=0$ is much simpler. We note that for all monomials $x \neq p q^{2}$ we have $\left(c_{1}-c_{3}\right)\left(p q^{2} \wedge x\right)=(\operatorname{deg}(x)+\mathrm{wg}(x)) \cdot x$. Since the monomial $p q^{2}$ cannot arise from $\tilde{c_{2}}(x \wedge y)$, we only need to consider

$$
\begin{aligned}
& M\left(\tilde{c_{2}}, c_{1}-c_{3}\right)\left(p q^{2} \wedge x \wedge y\right) \\
& \quad=(\operatorname{wg}(x)+\operatorname{deg}(x)+\operatorname{wg}(y)+\operatorname{deg}(y)) \tilde{c_{2}}(x \wedge y) \\
& \quad-\left(\operatorname{wg}\left(\tilde{c_{2}}(x \wedge y)+\operatorname{deg}\left(\tilde{c_{2}}(x \wedge y)\right)\right) \tilde{c_{2}}(x \wedge y)\right. \\
& \quad=-\left(\operatorname{wg}\left(\tilde{c_{2}}\right)+\operatorname{deg}\left(\tilde{c_{2}}\right)\right) \tilde{c_{2}}(x \wedge y)=0 .
\end{aligned}
$$

With this, the proof is complete. 
Case 2: $\mathbf{w}=3$. The cohomology space $H_{(-1,3)}^{2}\left(L_{1} ; L_{1}\right)$ is generated by $c_{4}, c_{5}$ and $c_{6}$, with

$$
\left\{\begin{array}{l}
c_{4}\left(q^{3} \wedge p^{k} q^{\ell}\right)=(k-\ell) p^{k} q^{\ell},(k, \ell) \neq(0,3), \\
c_{5}\left(p q^{2} \wedge p^{k} q^{\ell}\right)=-2 \ell p^{k+1} q^{\ell-1},(k, \ell) \neq(1,2), \\
c_{6}\left(q^{3} \wedge p^{k} q^{\ell}\right)=-(k+\ell-2) p^{k} q^{\ell},(k, \ell) \neq(0,3) .
\end{array}\right.
$$

Again, one sees that $c_{i}=\mathrm{d}\left(b_{i}\right)$, with

$$
b_{4}=q^{3} \mapsto p q, \quad b_{5}=p q^{2} \mapsto p^{2} \quad \text { and } \quad b_{6}=q^{3} \mapsto\left(p \partial_{p}+q \partial_{q}\right) .
$$

The Lie square of $c=\alpha_{4} c_{4}+\alpha_{5} c_{5}+\alpha_{6} c_{6}$ is trivial, but the Massey cube is trivial if and only if one of the following conditions is satisfied:

(1) $\alpha_{5}=0$,

(2) $-\alpha_{6}+3 \alpha_{5}-3 \alpha_{4}=0$,

(3) $-\alpha_{6}+6 \alpha_{5}-3 \alpha_{4}=0$.

A cocycle $c$ in the first plane, $\alpha_{5}=0$, has a trivial extension. This is similar to the case $\alpha_{2}=0$ before. The second plane, $-\alpha_{6}+3 \alpha_{5}-3 \alpha_{4}=0$, is spanned by $c_{4}-3 c_{6}$ (which is also in the first plane) and $c=c_{4}+c_{5}$. Instead of $c$ we take the new representative $\tilde{c}=\mathrm{d}(\tilde{b})$, with

$$
\tilde{b}=\sum_{k+\ell \geq 3} p^{k} q^{\ell} \mapsto\left[\frac{\ell}{2}\right] p^{k+1} q^{\ell-2},
$$

where $[x]$ denotes the floor function (entier) of $x$. We prove that $M(\tilde{c}, \tilde{c})=0$. The nonzero actions of $\tilde{c}$ are given by $\tilde{c}\left(p^{k} q^{\ell} \wedge p^{m} q^{n}\right)=(k(n-1)-\ell(m+1)) p^{k+m} q^{\ell+n-3}, \quad \ell$ even, $n$ odd, $\tilde{c}\left(p^{k} q^{\ell} \wedge p^{m} q^{n}\right)=\frac{1}{2}(2 k(n-1)+n-2 m(\ell-1)-\ell) p^{k+m} q^{\ell+n-3}, \quad n, \ell$ odd.

There are two cases to be considered, of which we only write out the first. This is the case with $\ell, n$ and $t$ odd in

$$
\begin{aligned}
& \frac{1}{2} M(\tilde{c}, \tilde{c})\left(p^{k} q^{\ell} \wedge p^{m} q^{n} \wedge p^{s} q^{t}\right) \\
& =(2 k(n-1)-2 m(\ell-1)+n-\ell) \tilde{c}\left(p^{k+m} q^{\ell+n-3} \wedge p^{s} q^{t}\right) \\
& +(2 m(t-1)-2 s(n-1)+t-n) \tilde{c}\left(p^{m+s} q^{n+t-3} \wedge p^{k} q^{\ell}\right) \\
& -(2 k(t-1)-2 s(\ell-1)+t-\ell) \tilde{c}\left(p^{k+s} q^{\ell+t-3} \wedge p^{m} q^{n}\right) \\
& =\frac{1}{2}\{(2 k(n-1)-2 m(\ell-1)+n-\ell) \\
& \cdot(2(k+m)(t-1)-2 s(\ell+n-4)+t-\ell-n+3) \\
& \quad+(2 m(t-1)-2 s(n-1)+t-n) \\
& \quad \cdot(2(m+s)(\ell-1)-2 k(n+t-4)+\ell-n-t+3) \\
& \quad-(2 k(t-1)-2 s(\ell-1)+n-\ell) \\
& \quad \cdot(2(k+s)(n-1)-2 m(\ell+t-4)+n-\ell-t+3)\} p^{k+m+s} q^{\ell+n+t-6} \\
& =0 . \quad
\end{aligned}
$$

The proof of $M\left(\tilde{c}, c_{4}-3 c_{6}\right)=0$ is similar to the proof of $M\left(\tilde{c_{2}}, c_{1}-c_{3}\right)=0$; it boils down to $\operatorname{wg}(\tilde{c})+3 \operatorname{deg}(\tilde{c})=0$. This completes the proof that the cocycles 
in the second plane define a global deformation by means of a trivial extension. One can easily check that the elements in the third plane also have a trivial extension.

There is a geometrical description for the deformations given by the cocycles that belong to the first or third plane. Let $\varphi: L_{1} \mapsto \overline{L_{0}}$ be $\varphi=$ id $-t b$, with $b=q^{3} \mapsto\left(\alpha_{4} p q+\alpha_{6}\left(p \partial_{p}+q \partial_{q}\right)\right)+\alpha_{5}\left(p q^{2} \mapsto p^{2}\right)$. Clearly, $\varphi$ defines a linear embedding of $L_{1}$ into $\overline{L_{0}}$. We demand $\varphi\left(L_{1}\right)$ to be a subalgebra in $\overline{L_{0}}$; this is a necessary and sufficient condition for $\varphi$ to be the realization mapping of an embedded deformation of $L_{1}$. There is only one commutator that needs to be checked:

$$
\begin{gathered}
{\left[\varphi\left(q^{3}\right), \varphi\left(p q^{2}\right)\right]=\left[q^{3}-\alpha_{4} t p q-\alpha_{6} t\left(p \partial_{p}+q \partial_{q}\right), p q^{2}-\alpha_{5} t p^{2}\right]} \\
=-3 q^{4}+t\left(-\alpha_{4}-\alpha_{6}+6 \alpha_{5}\right) p q^{2}-2 t^{2} \alpha_{4} \alpha_{5} p^{2} \in \varphi\left(L_{1}\right) .
\end{gathered}
$$

Hence, the compatibility condition is

$$
\alpha_{5}\left(-\alpha_{6}+6 \alpha_{5}-3 \alpha_{4}\right)=0 .
$$

Under this condition we get, by introducing $[x, y]_{t}=\varphi^{-1}([\varphi(x), \varphi(y)])$, an embedded deformation of $L_{1}$ with infinitesimal generator $c=\alpha_{4} c_{4}+\alpha_{5} c_{5}+$ $\alpha_{6} c_{6}$.

\section{Deformations of $L_{1}(W(2))$}

3.1. The cohomology space $H^{2}\left(L_{1} ; L_{1}\right)$. Here, $W(2)$ is the Lie algebra of polynomial vector fields on the plane. As in the previous section, we use the coordinates $p$ and $q$. A basis for $W(2)$ is $\left\{p^{k} q^{\ell} \partial_{p}, p^{k} q^{\ell} \partial_{q}, \quad k, \ell \geq 0\right\}$. On $W(2)$ there also exist two gradings; these are similar to the gradings of $H(2)$. The gradings are given by $\operatorname{deg}\left(p^{k} q^{\ell} \partial_{p}\right)=\operatorname{deg}\left(p^{k} q^{\ell} \partial_{q}\right)=k+\ell-1$, $\operatorname{wg}\left(p^{k} q^{\ell} \partial_{p}\right)=k-\ell-1$ and $\operatorname{wg}\left(p^{k} q^{\ell} \partial_{q}\right)=k-\ell+1$. Again, we define $L_{k}=L_{k}(W(2))$ to be the Lie subalgebra generated by the elements of degree greater than or equal to $k$, and we investigate homogeneous deformations of $L_{1}$.

Our first task is to compute $H_{(d, w)}^{2}\left(L_{1} ; L_{1}\right)$. From [6] we know that $H_{(d)}^{2}\left(L_{1}\right)$ $\neq 0$ can only occur if $d \in\{-2,-3\}$. Hence, by using the Feigin-Fuks spectral sequence, we conclude that $H_{(d, w)}^{2}\left(L_{1} ; L_{1}\right)=0$ for all $d<-2$. On account of the rigidity of $W(2)$ we do not expect that there are nontrivial deformations of positive degrees. So we only calculated $H_{(d, w)}^{2}\left(L_{1} ; L_{1}\right)$ for $d \leq 0$. Here is the result.

\section{Theorem 2. There holds}

$$
\operatorname{dim} H_{(d, w)}^{2}\left(L_{1} ; L_{1}\right)= \begin{cases}6, & d=-1, \quad w \in\{-1,1\}, \\ 4, & d=-1, \quad w \in\{-3,3\}, \\ 1, & d=-1, \quad w \in\{-5,5\}, \\ 0 & \text { in all other cases with } d \leq 0 .\end{cases}
$$

3.2. Global deformations. The cases of positive and negative weights convert to each other after interchanging $p$ and $q$. Therefore, we will only consider the cases with positive weights. 
Case 1: $\mathbf{w}=5$. The space $H_{(-1,5)}^{2}\left(L_{1} ; L_{1}\right)$ is generated by $c=\mathrm{d}(b)$, where $b \in C_{(-1,5)}^{1}\left(L_{1} ; L_{0}\right)$ is given by $b=q^{2} \partial_{p} \rightarrow p \partial_{q}$. The nonzero actions of $c$ are

$$
\left\{\begin{array}{l}
c\left(q^{2} \partial_{p} \wedge p^{k} q^{\ell} \partial_{p}\right)=p^{k} q^{\ell} \partial_{q}-\ell p^{k+1} q^{\ell-1} \partial_{p}, \quad(k, \ell) \neq(0,2), \\
c\left(q^{2} \partial_{p} \wedge p^{k} q^{\ell} \partial_{q}\right)=-\ell p^{k+1} q^{\ell-1} \partial_{q} .
\end{array}\right.
$$

Since $M(c, c)=0, c$ has a trivial extension. Again this can be geometrically interpreted. Define $\varphi: L_{1} \rightarrow L_{0}$ by $\varphi=\mathrm{id}-t b$; then $\varphi\left(L_{1}\right)$ is a subalgebra in $L_{0}$ and $[x, y]_{t}=\varphi^{-1}([\varphi(x), \varphi(y)])$ is the deformation given by $c$.

Case 2: $\mathbf{w}=3$. The cohomology space $H_{(-1,3)}^{2}\left(L_{1} ; L_{1}\right)$ is generated by $c_{i} \quad(1 \leq$ $i \leq 4)$ with

$$
\left\{\begin{array}{l}
c_{1}\left(p q \partial_{p} \wedge p^{k} q^{\ell} \partial_{p}\right)=p^{k} q^{\ell} \partial_{q}-\ell p^{k+1} q^{\ell-1} \partial_{p}, \quad(k, \ell) \neq(1,1), \\
c_{1}\left(p q \partial_{p} \wedge p^{k} q^{\ell} \partial_{q}\right)=-\ell p^{k+1} q^{\ell-1} \partial_{q} \\
c_{2}\left(q^{2} \partial_{q} \wedge p^{k} q^{\ell} \partial_{p}\right)=p^{k} q^{\ell} \partial_{q}-\ell p^{k+1} q^{\ell-1} \partial_{p}, \\
c_{2}\left(q^{2} \partial_{q} \wedge p^{k} q^{\ell} \partial_{q}\right)=-\ell p^{k+1} q^{\ell-1} \partial_{q}, \quad(k, \ell) \neq(0,2) \\
c_{3}\left(q^{2} \partial_{p} \wedge p^{k} q^{\ell} \partial_{p}\right)=-(k-1) p^{k} q^{\ell} \partial_{p},(k, \ell) \neq(0,2) \\
c_{3}\left(q^{2} \partial_{p} \wedge p^{k} q^{\ell} \partial_{q}\right)=-k p^{k} q^{\ell} \partial_{q}, \\
c_{4}\left(q^{2} \partial_{p} \wedge p^{k} q^{\ell} \partial_{p}\right)=-\ell p^{k} q^{\ell} \partial_{p},(k, \ell) \neq(0,2) \\
c_{4}\left(q^{2} \partial_{p} \wedge p^{k} q^{\ell} \partial_{q}\right)=-(\ell-1) p^{k} q^{\ell} \partial_{q} .
\end{array}\right.
$$

Again, $c_{i}=\mathrm{d}\left(b_{i}\right)$, where

$b_{1}=p q \partial_{p} \mapsto p \partial_{q}, b_{2}=q^{2} \partial_{q} \mapsto p \partial_{q}, b_{3}=q^{2} \partial_{p} \mapsto p \partial_{p} \quad$ and $\quad b_{4}=q^{2} \partial_{p} \mapsto q \partial_{q}$.

The Lie square of $c=\alpha_{1} c_{1}+\alpha_{2} c_{2}+\alpha_{3} c_{3}+\alpha_{4} c_{4}$ is trivial if

(1) $\alpha_{2}+2 \alpha_{1}=0$ or

(2) $2 \alpha_{4}-\alpha_{3}+\alpha_{2}-2 \alpha_{1}=0$.

In the second case the Massey cube is also trivial; however, in the first case there are additional constraints. There are two possibilities:

(1.i) $\alpha_{1}=\alpha_{2}=0$,

(1.ii) $2 \alpha_{4}-\alpha_{3}-2 \alpha_{1}=0$.

If these conditions are fulfilled (1.i or 1.ii or 2), then the fourth Massey power is also trivial. So there appear to be three options for integrable infinitesimal deformations. We will show that in all these cases there exists a cocycle with a trivial extension.

For case (1.i) and (2) there is a geometrical description. Define $\varphi: L_{1} \mapsto L_{0}$ by $\varphi=\mathrm{id}-t b$, with $b=\alpha_{1} b_{1}+\alpha_{2} b_{2}+\alpha_{3} b_{3}+\alpha_{4} b_{4}$, and demand $\varphi\left(L_{1}\right)$ to be a subalgebra in $L_{0}$. There are two compatibility conditions:

$$
\begin{aligned}
& {\left[\varphi\left(p q \partial_{p}\right), \varphi\left(q^{2} \partial_{p}\right)\right]=\left[p q \partial_{p}-\alpha_{1} t p \partial_{q}, q^{2} \partial_{p}-\alpha_{3} t p \partial_{p}-\alpha_{4} t q \partial_{q}\right]} \\
& \quad=-q^{3} \partial_{p}+t\left(\left(-2 \alpha_{1}+\alpha_{4}\right) p q \partial_{p}+\alpha_{1} q^{2} \partial_{q}\right)+t^{2} \alpha_{1}\left(-\alpha_{3}+\alpha \alpha_{4}\right) p \partial_{q} \in \varphi\left(L_{1}\right) .
\end{aligned}
$$

So, $\alpha_{1}\left(2 \alpha_{4}-\alpha_{3}+\alpha_{2}-2 \alpha_{1}\right)=0$. Next,

$$
\begin{aligned}
& {\left[\varphi\left(q^{2} \partial_{q}\right), \varphi\left(q^{2} \partial_{p}\right)\right]=\left[q^{2} \partial_{q}-\alpha_{2} t p \dot{\partial}_{q}, q^{2} \partial_{p}-\alpha_{3} t p \partial_{p}-\alpha_{4} t q \partial_{q}\right]} \\
& \quad=2 q^{3} \partial_{p}+t\left(\alpha_{4}+\alpha_{2}\right) q^{2} \dot{\partial}_{q}-2 t \alpha_{2} p q \dot{\partial}_{p}+t^{2} \alpha_{2}\left(-\alpha_{3}+\alpha_{4}\right) p \partial_{q} \in \varphi\left(L_{1}\right) .
\end{aligned}
$$


So, $\alpha_{2}\left(2 \alpha_{4}-\alpha_{3}+\alpha_{2}-2 \alpha_{1}\right)=0$. The compatibility conditions coincide with the conditions of (1.i) and (2), so these cases describe embedded deformations.

It is interesting to compare these results with the results for $L_{1}(H(2))$. To distinguish $L_{k}(H(2))$ from $L_{k}(W(2))$, we write $\mathbf{L}_{\mathbf{k}}$ for $L_{k}(H(2))$. Similarly, we write $\mathbf{b}_{i}$ for $b_{i}$ and $\boldsymbol{\alpha}_{i}$ for the $\alpha_{i}$ used in the previous section. If we restrict $\varphi$ to $\mathbf{L}_{\mathbf{1}}$, then we have $\tilde{\varphi}: \mathbf{L}_{\mathbf{1}} \mapsto \overline{\mathbf{L}_{\mathbf{0}}}$ given as in $\S 2.2$ with

$$
\alpha_{4}=\frac{3\left(\alpha_{3}-\alpha_{4}\right)}{2}, \quad \alpha_{5}=\frac{1}{2} \alpha_{2}-\alpha_{1}, \quad \alpha_{6}=\frac{-3\left(\alpha_{3}+\alpha_{4}\right)}{2} .
$$

From $\S 2.2$ we know that there are two possibilities for $\tilde{\varphi}$ :

(1) $\alpha_{5}=0$. This is just the restriction of $\varphi$ with $\alpha_{1}=\alpha_{2}=0$.

(2) $-\alpha_{6}+6 \alpha_{5}-3 \alpha_{4}=3\left(2 \alpha_{4}-\alpha_{3}+\alpha_{2}-2 \alpha_{1}\right)=0$. This is the restriction of $\varphi$ in the other case.

Hence, the embedded deformations of $\mathbf{L}_{1}$ of degree -1 and weight 3 are just the restrictions of the embedded deformations of $L_{1}$.

The third case $\left(\alpha_{2}+2 \alpha_{1}=0,2 \alpha_{4}-\alpha_{3}-2 \alpha_{1}=0\right)$ is a plane which is spanned by $2 c_{3}+c_{4}$ and $c=-\frac{1}{2} c_{1}+c_{2}+\frac{1}{3} c_{3}-\frac{1}{3} c_{4}$. Instead of $c$ we take the representative $\tilde{c}=\mathrm{d}(\tilde{b})$, with

$$
\begin{aligned}
\tilde{b}= & \sum_{\substack{k+\ell \geq 3 \\
\ell \text { even }}} p^{k} q^{\ell-1} \partial_{p} \mapsto\left(-\frac{1}{2} p^{k} q^{\ell-2} \partial_{q}+\frac{1}{2}(\ell-2) p^{k+1} q^{\ell-3} \partial_{p}\right) \\
& +\sum_{\substack{k+\ell \geq 3 \\
\ell \text { odd }}} p^{k} q^{\ell-1} \partial_{p} \mapsto \frac{\left[\frac{\ell}{2}\right]}{\ell+2 k}\left(-p^{k} q^{\ell-2} \partial_{q}+(\ell-2+2 k) p^{k+1} q^{\ell-3} \partial_{p}\right) \\
& +\sum_{\substack{k+\ell \geq 2 \\
\ell \text { even }}} p^{k} q^{\ell} \partial_{q} \mapsto \frac{\ell}{2} p^{k+1} q^{\ell-2} \partial_{q} \\
& +\sum_{\substack{k+\ell \geq 2 \\
\ell \text { odd }}} p^{k} q^{\ell} \partial_{q} \mapsto \frac{1}{(\ell+2 k+2)} \\
& \times\left(2(\ell-1) p^{k+2} q^{\ell-3} \partial_{p}+\left[\frac{\ell}{2}\right] \frac{((k+2)(\ell+2 k+2)-\ell)}{(k+1)} p^{k+1} q^{\ell-2} \partial_{q}\right) .
\end{aligned}
$$

This $\tilde{c}$ satisfies $M(\tilde{c}, \tilde{c})=0$ and $M\left(\tilde{c}, 2 c_{3}+c_{4}\right)=0$. Hence, the cocycles in this plane also define a global deformation by means of a trivial extension. If we restrict $\tilde{c}=\mathbf{d}(\tilde{b})$ to $\mathbf{L}_{\mathbf{1}}$, then we obtain $\tilde{\mathbf{c}}=\mathrm{d}(\tilde{\mathbf{b}})$ from case 2 in $\S 2.2$. The restriction of $\frac{2}{3}\left(2 c_{3}+c_{4}\right)$ equals $\mathbf{c}_{4}-3 \mathbf{c}_{6}$ (see $\left.\S 2.2\right)$. So, the deformations of $\mathbf{L}_{\mathbf{1}}$ given by the second plane in case 2 in $\S 2.2$ arise by restricting the global deformations of $L_{1}$ of this plane to $\mathbf{L}_{1}$. Hence, all global deformations of $\mathbf{L}_{1}$ of weight 3 are induced by global deformations of $L_{1}$. 
Case 3: $\mathbf{w}=1$. The cohomology space $H_{(-1,1)}^{2}\left(L_{1} ; L_{1}\right)$ is generated by $c_{i}(1 \leq$ $i \leq 6)$ with

$$
\begin{cases}c_{1}\left(p^{2} \partial_{p} \wedge p^{k} q^{\ell} \partial_{p}\right)=p^{k} q^{\ell} \partial_{q}-\ell p^{k+1} q^{\ell-1} \partial_{p},(k, \ell) \neq(2,0) \\ c_{1}\left(p^{2} \partial_{p} \wedge p^{k} q^{\ell} \partial_{q}\right)=-\ell p^{k+1} q^{\ell-1} \partial_{q} \\ c_{2}\left(p q \partial_{q} \wedge p^{k} q^{\ell} \partial_{p}\right)=p^{k} q^{\ell} \partial_{q}-\ell p^{k+1} q^{\ell-1} \partial_{p} \\ c_{2}\left(p q \partial_{q} \wedge p^{k} q^{\ell} \partial_{q}\right)=-\ell p^{k+1} q^{\ell-1} \partial_{q},(k, \ell) \neq(1,1) \\ c_{3}\left(p q \partial_{p} \wedge p^{k} q^{\ell} \partial_{p}\right)=-(k-1) p^{k} q^{\ell} \partial_{p}, \quad(k, \ell) \neq(1,1) \\ c_{3}\left(p q \partial_{p} \wedge p^{k} q^{\ell} \partial_{q}\right)=-k p^{k} q^{\ell} \partial_{q} \\ c_{4}\left(q^{2} \partial_{q} \wedge p^{k} q^{\ell} \partial_{p}\right)=-(k-1) p^{k} q^{\ell} \partial_{p}, \\ c_{4}\left(q^{2} \partial_{q} \wedge p^{k} q^{\ell} \partial_{q}\right)=-k p^{k} q^{\ell} \partial_{q},(k, \ell) \neq(0,2) \\ c_{5}\left(p q \partial_{p} \wedge p^{k} q^{\ell} \partial_{p}\right)=-\ell p^{k} q^{\ell} \partial_{p},(k, \ell) \neq(1,1) \\ c_{5}\left(p q \partial_{p} \wedge p^{k} q^{\ell} \partial_{q}\right)=-(\ell-1) p^{k} q^{\ell} \partial_{q}, \\ c_{6}\left(q^{2} \partial_{q} \wedge p^{k} q^{\ell} \partial_{p}\right)=-\ell p^{k} q^{\ell} \partial_{p} \\ c_{6}\left(q^{2} \partial_{q} \wedge p^{k} q^{\ell} \partial_{q}\right)=-(\ell-1) p^{k} q^{\ell} \partial_{q}, \quad(k, \ell) \neq(0,2)\end{cases}
$$

Again, $c_{i}=\mathrm{d}\left(b_{i}\right)$ with

$$
\begin{gathered}
b_{1}=p^{2} \partial_{p} \mapsto p \partial_{q}, \quad b_{2}=p q \partial_{q} \mapsto p \partial_{q}, \quad b_{3}=p q \partial_{p} \mapsto p \partial_{p}, \\
b_{4}=q^{2} \partial_{q} \mapsto p \partial_{p}, \quad b_{5}=p q \partial_{p} \mapsto q \partial_{q} \quad \text { and } \quad b_{6}=q^{2} \partial_{q} \mapsto q \partial_{q} .
\end{gathered}
$$

The seventh candidate, $\mathrm{d}\left(q^{2} \partial_{p} \mapsto q \partial_{p}\right)$ is linearly dependent in $H_{(-1,1)}^{2}\left(L_{1} ; L_{1}\right)$. This can be seen by taking $\alpha=1 \mapsto \partial_{q}$ and computing

$$
\begin{aligned}
\mathrm{d}(\alpha)= & b_{3}+b_{2}+2 b_{6}+2\left(q^{2} \partial_{p} \mapsto q \partial_{p}\right) \\
& +\sum_{k+\ell \geq 3} \ell\left(p^{k} q^{\ell} \partial_{p} \mapsto p^{k} q^{\ell-1} \partial_{p}+p^{k} q^{\ell} \partial_{q} \mapsto p^{k} q^{\ell-1} \partial_{q}\right) .
\end{aligned}
$$

By applying $d$ again, we obtain

$$
c_{3}+c_{2}+2 c_{6}+2 \mathrm{~d}\left(q^{2} \partial_{p} \mapsto q \partial_{p}\right) \in B_{(-1,1)}^{2}\left(L_{1} ; L_{1}\right) .
$$

For the combination $c=\alpha_{1} c_{1}+\alpha_{2} c_{2}+\alpha_{3} c_{3}+\alpha_{4} c_{4}+\alpha_{5} c_{5}+\alpha_{6} c_{6}$ we calculated the obstructions. There are three possibilities for which the Lie square and Massey cube are trivial:

(1) $\alpha_{1}=\alpha_{2}=0$ and $\alpha_{3} \alpha_{6}=\alpha_{5} \alpha_{4}$;

(2) $\alpha_{1}=2 \alpha_{2}, \alpha_{4}=2 \alpha_{3}, \alpha_{5}=\alpha_{2}$ and $\alpha_{6}=2 \alpha_{2}$;

(3) $\alpha_{1}=\alpha_{5}=\alpha_{6}=0$.

It appears that in all three cases there exist cocycles that define global deformations of $L_{1}$ by means of a trivial extension. The cases (1) and (2) are the compatibility conditions for $\varphi: L_{1} \mapsto L_{0}$ given by $\varphi=$ id $-t b$ with $b=\alpha_{1} b_{1}+\alpha_{2} b_{2}+\alpha_{2} b_{2}+\alpha_{3} b_{3}+\alpha_{4} b_{4}+\alpha_{5} b_{5}+\alpha_{6} b_{6}$. So in these cases we have again embedded deformations. We restrict $\varphi$ to $\mathbf{L}_{1}$; then we have $\tilde{\varphi}: \mathbf{L}_{1} \mapsto \overline{\mathbf{L}_{0}}$ as in $\S 2.2$, with

$$
\boldsymbol{\alpha}_{1}=\frac{1}{2}\left(2 \alpha_{3}-\alpha_{4}-2 \alpha_{5}+\alpha_{6}\right), \quad \alpha_{2}=\frac{1}{2}\left(2 \alpha_{2}-\alpha_{1}\right),
$$




$$
\alpha_{3}=\frac{1}{2}\left(-2 \alpha_{3}+\alpha_{4}-2 \alpha_{5}+\alpha_{6}\right) \text {. }
$$

In both cases, $\alpha_{2}=0$. This is precisely the compatibility condition of $\S 2.2$. It is obvious that the restriction of the deformations of case (2) are not interesting, since $\alpha_{1}=\alpha_{2}=\alpha_{3}=0$. The deformations of $\mathbf{L}_{1}$ given by the first plane (see case 1 in $\S 2.2$ ) arise as restrictions of the deformations of $L_{1}$ described by (1).

In the third case $\left(\alpha_{1}=\alpha_{5}=\alpha_{6}=0\right)$, there is a three-dimensional space of cocycles spanned by $c_{3}, c_{4}$ and $c=c_{2}-c_{3}-2 c_{4}$. Instead of $c$ we take the representative $\tilde{c}=\mathrm{d}(\tilde{b})$, with

$$
\begin{aligned}
\tilde{b}= & -2 \sum_{n \geq 0} q^{n+2} \partial_{q} \mapsto p q^{n} \partial_{p}+\sum_{n \geq 2}(n-1)\left(q^{n+1} \partial_{q} \mapsto q^{n} \partial_{q}+q^{n+1} \partial_{p} \mapsto q^{n} \partial_{p}\right) \\
& +\sum_{\substack{n \geq 0 \\
m \geq 1}}(n+1)\left(p^{m} q^{n+1} \partial_{q} \mapsto p^{m} q^{n} \partial_{q}\right)+\sum_{n \geq 0}(n-1)\left(p q^{n+1} \partial_{p} \mapsto p q^{n} \partial_{p}\right) \\
& +\sum_{\substack{n \geq 0 \\
m \geq 2}}(n+1)\left(p^{m} q^{n+1} \partial_{p} \mapsto p^{m} q^{n} \partial_{p}\right) .
\end{aligned}
$$

The products $M(\tilde{c}, \tilde{c}), \quad M\left(\tilde{c}, c_{3}\right), \quad M\left(\tilde{c}, c_{4}\right), \quad M\left(c_{3}, c_{3}\right), \quad M\left(c_{3}, c_{4}\right)$ and $M\left(c_{4}, c_{4}\right)$ are 0 , so we have a three-dimensional space of cocycles that define global deformations by means of a trivial extension. We can restrict these deformations to $\mathbf{L}_{\mathbf{1}}$. The restriction of $\tilde{b}$ equals $\tilde{\mathbf{b}}_{2}$ of case 1 in $\S 2.2$, and the restriction of $\alpha_{3} c_{3}+\alpha_{4} c_{4}$ equals $\frac{1}{2}\left(2 \alpha_{3}-\alpha_{4}\right)\left(\mathbf{c}_{1}-\mathbf{c}_{3}\right)$. Hence, the deformations of $\mathbf{L}_{1}$ corresponding to the special plane given in case (1) are also just the restriction of deformations of $L_{1}$.

\section{Conclusion}

We determined all infinitesimal deformations of $L_{1}(H(2))$ and $L_{1}(W(2))$, and moreover investigated which of the homogeneous infinitesimal deformations can be integrated. In case that it can be extended, we gave a possible extension. The question arises, whether to any infinitesimal deformation there are two nonequivalent extensions. As said before in $\S 1$, this can happen if at some point in the two extending series $\left(\varphi_{2}, \varphi_{3}, \ldots\right)$ and $\left(\varphi_{2}, \varphi_{3}, \ldots, \varphi_{i-1}\right.$, $\left.\varphi_{i}^{\prime}, \varphi_{i+1}^{\prime}, \ldots\right), \varphi_{i}$ and $\varphi_{i}^{\prime}$ are noncohomologous. Since we assume deformations to be homogeneous, we have that $\operatorname{deg}\left(\varphi_{i}\right)=i \operatorname{deg}\left(\varphi_{1}\right)$. Hence we car conclude that the deformations of $L_{1}(W(2))$ are unique (up to a transformation) since $H^{2}\left(L_{1}(W(2)) ; L_{1}(W(2))\right.$ is only nonzero in degree -1 . For $L_{1}(H(2))$ there could be branching of the deformation in the term $\varphi_{2}$, since $H^{2}\left(L_{1}(H(2)) ; L_{1}(H(2))\right.$ is nonzero at degree $(-1$ and $)-2$. Comparing weights, one sees that only for degree -1 and weight 1 can there be problems. However, one can prove that a term $\varphi_{2}$ which is nonzero in $H^{2}\left(L_{1}(H(2)) ; L_{1}(H(2))\right.$ leads to a real obstruction.

In conclusion, we can state that the constructed homogeneous deformations of $L_{1}(H(2))$ and $L_{1}(W(2))$ are indeed all homogeneous deformations of negative degree. In this sense, this study is unique. 


\section{BIBLIOGRAPHY}

1. A. Fialowski, On the deformations of $L_{1}$, Studia Sci. Math. Hungar. 20 (1985), 433-438.

2. Yu. Yu. Kochetkov and G.F. Post, Deformations of the nilpotent infinite-dimensional Lie algebra $L_{2}$, Functional Anal. Appl. 26 (1992), 304-305.

3. N. van den Hijligenberg, Deformations of nilpotent parts of the Neveu-Schwarz and Ramond superalgebras, J. Math. Phys. 35 (1994), 1427-1440.

4. B. L. Feigin and D. B. Fuks, Homology of the Lie algebra of vector fields on the line, Functional Anal. Appl. 14 (1980), 201-212.

5. N. van den Hijligenberg and G. Post, Defining relations for Lie algebras of vector fields, Indag. Math. (N.S.) 2 (1991), 207-218.

6. D.B. Fuchs, Cohomology of infinite-dimensional Lie algebras, Plenum Press, New York, 1986.

CWI, P.O. Box 4079, 1009 AB Amsterdam, The NetherLands

E-mail address: nico@cwi.nl

Department of Applied Mathematics, University of Twente, P.O. Box 217, 7500 AE ENSCHEDE, THE NETHERLANDS

Current address, Y. Kotchetkov: Institute of New Technologies in Education, Moscow, Russia 